\title{
EDUCACIÓN
}

\section{Lineamiento pedagógico vigente en carreras de administración de empresas en una universidad privada de Asunción}

\author{
Gloria Concepción Martínez Pasmor ${ }^{1}$, Luis Fermín Zavala Reyna
}

\begin{abstract}
Resumen
Introducción: La presente investigación surge del cambio que se da en la educación tradicional. Cambio en el cual se percibe nuevos modelos de educación basada en competencias, cuyo modelo educativo está centrado en el estudiante y en el enriquecimiento de sus formas de aprendizaje, mediante diversas estrategias que le permiten adquirir el dominio de conocimientos, habilidades, actitudes, capacidades y valores para que su educación sea permanente a lo largo de toda su vida.
\end{abstract}

Objetivo: Analizar lineamientos pedagógicos vigentes en carreras de administración de empresas para la promoción de formulación de modelo de currículo basado en enfoque por competencias laborales en una Universidad privada de Asunción.

Material y Método: El diseño de investigación es no experimental, tipo transversal, descriptivo; está orientado a la descripción del fenómeno tal como se presenta en el momento de realizarse el estudio. El enfoque de la investigación es cuantitativo. Para la recogida de datos se recurrió a fuentes secundarias para la fundamentación del marco teórico y a fuentes primarias para la recolección de información de fuentes directas. La técnica de recolección de datos es la encuesta, cuestionario. La población la conforman egresados de la carrera de Administración de Empresas del periodo 2009- 2011. El muestreo probabilístico, aleatorio simple, con muestra representativa por estrato.

El procedimiento fue una sucesión de acciones de revisión de las literaturas, elaboración, validación y aplicación del instrumento, tratamiento estadístico de los datos y elaboración y presentación de propuestas.

Resultados: En los resultados se observa que en cuanto al Nivel de Aplicación de las competencias los egresados han recibido algunas de las competencias requeridas, tales como habilidades básicas de manejo del ordenador; conocimientos básicos de la profesión; habilidades de la gestión de la información; trabajo en equipo; preocupación por la calidad, entre otros.

\footnotetext{
1. Universidad María Auxiliadora (UMAX).

El trabajo de investigación corresponde a una Tesis para la obtención del título de Maestría.

E-mail:gmartinez@umax.edu.py
}

DOI: 10.26885/rcei.foro.2018.62 


\section{Lineamiento pedagógico vigente en carreras de administración de empresas. Martínez \& Zavala}

Además, los resultados enfatizan también en la importancia de competencias que deben poseer los egresados, resultando dentro de los cinco primeros lugares la toma de decisiones, capacidad de trabajar con un equipo multidisciplinario, capacidad de adaptarse a nuevas situaciones, resolución de problemas y compromiso ético.

Conclusiones: En la Universidad estudiada se descubre que aún sigue primando la educación tradicional que enfatiza en los saberes conceptuales y en las aéreas de conocimiento especializadas, que responden parcialmente a las necesidades actuales del mundo contemporáneo. Sin embargo, el currículo y los métodos de enseñanza tienen que ser flexibles para adaptarse a las necesidades y características de los estudiantes y de los diversos contextos sociales y culturales.

Se propone un diseño curricular basado en competencias para responder a los requerimientos de las empresas y sus actuales necesidades, para alcanzarlo se requiere en primer lugar, contar la decisión de cambiar la cultura académica y administrativa de la Universidad, así como la participación activa de sus directivos y docentes.

Posteriormente se debe realizar la clasificación de actividades por afinidad y enmarcarlos en los aprendizajes que corresponden a los saberes cognitivos (saber conocer), saberes procedimentales (saber hacer) y saberes actitudinales (saber ser), para construir el currículo por competencias. Y finalmente capacitar a los docentes en principios, implicancias y herramientas del enfoque de competencia, aspectos fundamentales e indispensables para su implementación.

Palabras clave: pedagogía, competencia, currículo.

\section{Referencias}

Arias Tibaquirá, A. P. (2011). Lineamientos para el diseño del perfil del Administrador de empresas de la Universidad de la sede Manizales: basado en un enfoque por competencias laborales (Tesis de Maestría). Universidad Nacional de Colombia. Manizales, Colombia.

Barron Tirado, M. C. (2009). Docencia universitaria y competencias didácticas. Perfiles Educativos, 31(125), p. 76-87.

Beneitone, P. et al. (2007). Reflexiones y perspectivas de la educación superior en América Latina: Informe final proyecto Tunning, 2004-2007. Bilbao: Universidad Deusto.

Bunk, G. P. (1994). La transmisión de las competencias en la formación y perfeccionamiento profesionales de la RFA. Revista Europea. Formación profesional, p. 8-14.

Díaz Villa, M. (2002). Flexibilidad Curricular en la Educación Superior en Colombia. Colombia: ICFES. 